\title{
Böckenförde 2.0: Offene Gesellschaften und autokratische Experimente
}

\author{
Stefan Brunnhuber
}

\section{Das Böckenförde-Paradox und seine Rezeption}

Der Verfassungsrechtler Ernst Wolfgang Böckenförde (19.9.1930 - 24.2.2019) formulierte erstmals im Jahr 1967 ein nach ihm benanntes Paradox: Offene Gesellschaften leben von Voraussetzungen, welche sie selbst nicht herstellen können. Es sind autonome, kritisch denkende und freie Bürger, ein kritischer öffentlicher Diskurs, die Fähigkeit Widersprüche und Meinungsverschiedenheiten durch gewählte Institutionen zu vermitteln, eine kritische und unabhängige Presse, ein von Traditionen und Parteien unabhängiger Wissenschaftsbetrieb, eine freie Religionsausübung sowie ein am individuellen Potential einzelner Mitglieder ausgerichtetes Bildungssystem, welches uns offene gesellschaftliche Verhältnisse ermöglicht - allesamt Voraussetzungen, welche die Verfassung einer Offenen Gesellschaft ohne Rechtszwang aus sich heraus nicht schaffen kann. Mehr noch, jede Art von autoritärem Gebot würde gerade diesen Kata$\log$ an Bedingungen der Freiheit, um dessen Willen ihre Mitglieder eine freiheitliche Grundordnung wählen, im Kern widersprechen und das Gesamtanliegen offener gesellschaftlicher Verhältnisse ad absurdum führen. ${ }^{1}$

Wirkungsgeschichtlich waren die Folgejahre um gerade jenes Paradox vor allem geprägt durch die Stellung der Religion im Aufbau eines säkularen Staates, die Bedeutung des Sozialkapitals als Bedingung einer Offenen Gesellschaft sowie die Gefahren einer grundrechtskonformen Aufkündigung von inter-generationellen Verpflichtungen. So wurde etwa deutlich, dass die Trennung von Staat und Religion ein relativ spätes und zugleich geographisch eher seltenes Phänomen darstellt. Buddhismus, Hinduismus, Islam, Taoismus, Konfuzianismus, aber auch die Orthodoxe Kirche haben häufig eine sehr enge Verbindung mit staatlichen Institutionen, öffentlichen Normen und den Gesetzen eines Landes. Selbst in westlichen Gesetzgebungsverfahren ist nicht nur auf einer semantischen Ebene der Bezug zum Christentum oder zumindest zu einer monotheistischen Religion immer wieder zu spüren und auch nachzulesen. Offene Gesellschaften sind in diesem Sinne nicht völlig säkularisierte Gesellschaften. Aber Offene Gesellschaften kennen den Unterschied zwischen dem was säkular und dem was spirituell ist. ${ }^{2}$ So wurde, gestützt durch empirische Untersuchungen des Weiteren sichtbar, dass eine freie und heterodoxe Religionsausübung innerhalb eines säkularen Staates zwar ein Faktor für den Aufbau des Sozialkapitals darstellt, aber dass in gleicher Weise

\footnotetext{
${ }^{1}$ Böckenförde, Die Entstehung des Staates als Vorgang der Säkularisation, FS Buve, 1967, 75-94.

${ }^{2}$ Schlaich, Konfessionalität - Säkularität - Offenheit. Der christliche Glaube und der freiheitlichdemokratische Verfassungsstaat, FS Rendtorff, 1985, 175-198; Busse, Das Prinzip staatlicher Neutralität und die Freiheit der Religionsausübung: Eine Analyse der Rechtsprechung zum ethisch-religiösen Neutralitätsgebot, 2013, 216.
} 
auch andere nicht religiös motivierte Faktoren, wie reziproke Toleranz und Pluralismus, Vertrauen, Solidarität und Verantwortung in ähnlicher Weise für den Bestand einer Offenen Gesellschaft konstitutiv sind. ${ }^{3}$ Schließlich wäre es denkbar, dass eine zukünftige Generation sich innerhalb des Verfassungsrahmens für andere Werte, etwa immaterielle Werte entscheidet und damit den inter-generationellen Konsens, zumindest in Teilen, wieder aufkündigt. Beispielsweise wäre es angesichts der zu erwartenden Klimaveränderungen kritisch-rational auf weiteres exponentielles Wachstum zu verzichten, einen geringeren materiellen Lebensstil zu wählen und damit die Gegenfinanzierung unserer sozialen Sicherungssysteme in der bisherigen Form aufzukündigen oder zumindest in Frage zu stellen. Und dies alles innerhalb einer verfassungskonformen Auseinandersetzung um zukünftige offene gesellschaftliche Verhältnisse. Die Fragen nach den Bedingungen und Voraussetzungen einer Offenen Gesellschaft bleiben folglich weiterhin kontrovers, auch wenn nicht weniger substantiell. ${ }^{4}$

\section{Böckenförde 2.0 im Zeitalter autokratischer Regime}

Nach dem vermeintlichen Ende der Geschichte ${ }^{5}$ stehen sich nun erneut Offene Gesellschaften und Autokratien als zwei Zivilisationsentwürfe entgegen. Empirisch lebt circa die Hälfte der Menschheit in politischen Systemen, welche durch eine Alleinherrschaft einzelner Personen, Clans oder einer Parteiorganisation charakterisiert sind, falls sie nicht gar innerhalb von failed states leben. Die andere Hälfte der Menschheit lebt in mehr oder weniger offenen, säkularen, demokratischen und rechtsstaatlichen Verhältnissen. Hinzu kommt, dass fast 1/4 der Mitglieder ihrer eigenen offenen Verfassung gegenüber kritisch eingestellt sind bzw. empirisch zwischen 30-44 \% eine eher autokratische Reaktionsbereitschaft besitzen. Die bedeutet in diesem Zusammenhang, dass immer dann, wenn die Kontrolle und Selbstwirksamkeit für den Bürger verloren zu gehen scheint, politischer Stabilität, ökonomischer Prosperität, sozialer Kohärenz und Zugehörigkeit ein höheres konstitutionelles Gewicht beigemessen wird als der freien politischen Partizipation sowie gesteigerter Risiko- und Veränderungsbereitschaft. ${ }^{6}$ Insbesondere globale Herausforderungen - Finanzmärkte, Digitalisierung, Klimakatastrophe, Verlust an Biodiversität, Zunahme der Einkommens- und Vermögensunterschiede - können dabei als Trigger dienen, anderen staatlichen Verfassungen, vorrangig autokratischen, eine höhere politische Kompetenz zuzusprechen als der eigenen, eher freiheitlich-demokratischen Grundordnung. Dies wird vor allem mit einem defizitären Konfliktmanagement und Problemlösungsverhalten in Verbindung gebracht, sowie mit mangelnder ökonomischer Prosperität und ungleichmäßiger Beteiligung an der Wohlstandsentwicklung. Diese erneute Systemkonkurrenz unterschiedlicher Verfassungsformen, welche mit dem Jahr 1989 als überwunden galt und im Washingtoner Konsens ${ }^{7}$

\footnotetext{
${ }^{3}$ Putnam, Bowling alone. America's declining social capital, Journal of Democracy 6/1 (1995) 65-78; van de Meer/Tolsma, Ethnic diversity and its effects on social cohesion, Annual review of sociology 40 (2014), 459-478.

${ }^{4}$ Kirchhof, Die kulturellen Voraussetzungen der Freiheit: Verfassungsrechtliche Überlegungen zur Wirtschaftsfreiheit, zur Forschungsfreiheit und zur Willensbildung in der Demokratie, 1995, 16.

${ }^{5}$ Fukuyama, The End of History and the last Man, 1992, 448.

${ }^{6}$ Stenner, The Authoritarian Dynamic, 2010, 123; Edsall, The contract with authoritarianism, New York Times v. 5.4.2018.

${ }^{7}$ Williamson, Washington Consensus, 2003, 25.
} 
von 1992 durch ein Mehr an Liberalisierung, Deregulierung und Privatisierung eine einseitig westliche Prägung erfuhr, tritt nun am beginnenden 21. Jahrhundert im neuen Gewand der Autokratien auf die Weltbühne. Inglehart spricht in diesem Zusammenhang gar von „dem ernstesten demokratischen Rückschritt seit 1930“. ${ }^{8}$

Vor diesem Hintergrund lässt sich in Anlehnung an das Böckenförde-Paradox gleichsam eine Version zweiter Ordnung formulieren: Autokratische Verfassungen leben selbst von Voraussetzungen, welche sie immanent nicht hinreichend einlösen können und funktionieren auf lange Sicht nur, weil es offene, freiheitliche und rechtsstaatliche Verfassungen gibt, welche gleichsam parallel zu ihnen existieren und in der Systemkonkurrenz auf lange Sicht dann doch einen Wettbewerbsvorteil genießen. Diesem Böckenförde-Paradox zweiter Ordnung möchte ich im Folgenden nachgehen.

\section{Autokratische Experimente und warum sie selbstlimitierend sind}

Von Autokratien spricht man, wenn die Staatsgewalt uneingeschränkt und unkontrolliert einigen wenigen oder einem Herrscher allein zur Verfügung steht. Man unterscheidet dabei kommunitäre Autokratien (China) mit einem Einparteiensystem, paternalistische Autokratien (Russland) mit einer stärkeren Betonung von Personen gegenüber Institutionen, sowie tribale Autokratien (Golfstaaten) mit ausgeprägten erbrechtlich verbindlichen Familienbanden. Für alle gilt, dass Sicherheit und ökonomischer Wohlstand wichtiger als politische Mitbestimmung und die Zustimmungswerte in der Bevölkerung für gerade jene politischen Verfassungen in der Regel höher sind als in westlichen Demokratien. Und für alle drei Spielarten gilt zudem, dass die für Offene Gesellschaften typischen Merkmale einer innovativen, kreativen und risikofreudigen Mittelschicht fehlen und an ihre Stelle ein hoher Anteil an system-stabilisierenden, eher reaktionären Funktionären tritt. Derzeit leben circa 3,3 Mrd. Menschen in über 58 Staaten unter autokratischen Regimen. Dabei sind die Ergebnisse mancher autokratischer Regime beeindruckend. Rasche, kurze Entscheidungswege, keine langwierigen und kontroversen Debatten, Millionen von Menschen werden umgesiedelt, aus Armut befreit, in Erziehungs- und Bildungseinrichtungen eingebracht und eine öffentliche Infrastruktur großen Ausmaßes geschaffen. Menschen genießen hier zwar keine politische Partizipation im westlichen Sinne, dafür aber ökonomische Prosperität und Stabilität. Selbst der dort ansässige Wissenschaftsbetrieb, der Umfang an Patentanmeldungen sowie der unternehmerische Erfolg einzelner internationaler Großbetriebe sprechen für sich. Diese Vorteile gelten jedoch nur auf den ersten Blick. Bei Licht betrachtet sind jene Vorteile selbstlimitierend und dependent.

Autokratien sind selbstlimitierend, weil sie kollabieren, wenn sie sich wirklicher Kritik aussetzen, weil sie stärker im Kopieren als in kreativer Auseinandersetzung sind. Sie sind selbstlimitierend und dependent, weil sie mehr auf Kontrolle als auf soziale und individuelle Kreativität setzen und weil sie auf Wissen zurückgreifen müssen, welches sie selbst nicht hinreichend generieren können, sondern nur aus Offenen Gesellschaften übernehmen können. Der vermeintliche Vorsprung stellt sich dann schnell als die zweitbeste Lösung heraus. Preise entstehen nur auf freien Märkten, Kreativität, individuelle Risikobereitschaft und Innovationsfähigkeit nur durch freie Menschen. Kritische Dis-

\footnotetext{
${ }^{8}$ Inglehart, The age of insecurity. Can Democracy Save itself?, Foreign Affairs v. 16.4.2018.
} 
kurse und eine freie Presse, welche den Unterschied zwischen Fakes und Fakten herstellen, gibt es nur dort, wo Menschen in Eigenverantwortung und kritischer Selbstdisziplin nach der Wahrheit suchen. Wirkliches Sozialkapital, welches Gesellschaften im Kern zusammenhält und auf reziproker Verantwortung und Vertrauen beruht, gibt es nur dort, wo Individuen freiwillig, autonom und sich auf Augenhöhe auf andere einlassen und sich gegenseitig vertrauen. Es muss ständig gepflegt werden und wird im autokratischen Experiment ersetzt durch eine externe Kontrolle über das, was richtig und falsch und was angemessen ist. Damit geht aber seine ursprüngliche Funktion verloren. ${ }^{9}$ Und es ist schließlich die Erziehung zu eigenständigem Denken, kritischer Urteilskraft, Fehlerfreundlichkeit, Respekt und Achtung, dem gegenseitigen Eingeständnis, dass wir alle viel zu wenig wissen, unsere Entscheidungen immer revisionsoffen bleiben müssen und auf einer dauerhaft unvollständigen Informationsbasis beruhen, welche uns als Spezies Mensch allein zur Verfügung steht, um Probleme zu lösen. ${ }^{10}$ Denn dies ist bei autoritären Entscheidungen einer politischen Nomenklatura, eines nicht-demokratischen Parteiapparats oder eines Familienclans nicht grundsätzlich anders als in einem öffentlich geführten, angstfreien Diskurs um den richtigen Weg. Selbst der Wissenschaftsbetrieb braucht für wahrhaftige Einblicke und ein tieferes Verständnis von Natur und Gesellschaft einen offenen, selbstkritischen und von Tradition, Partei und Religion unabhängigen Diskurs. All dies können Autokratien aus sich heraus nicht leisten.

Zugegebenermaßen können solche Ordnungen der Freiheit immer wieder missbraucht werden und man kann auch konstatieren, dass das überlegene Ordnungsmodell einer Offenen Gesellschaft nach 1989 keine hinreichende innere Weiterentwicklung antrat, um die Herausforderungen des 21. Jahrhunderts zu meistern. Aber dennoch gilt: Sie werden in ihrer grundlegenden Form nur in Offenen Gesellschaften geschaffen. Kontrolliertes Herrschaftswissen bedarf Offener Gesellschaften, um überhaupt regieren zu können. In diesem Sinne sind sie selbstlimitierend und dependent. Eine Welt, in der es nur Autokratien gäbe, wäre nicht nur ein Rückfall in geschlossene Verhältnisse, es wäre auch ein Verlust an Freiheit und Ordnung und zugleich ein freiwilliger Verzicht auf die für uns Menschen alleinig zur Verfügung stehenden Ressourcen zur Lösung von Problemen: Der selbstkritische und autonome Verstand, welcher um seine eigenen Grenzen Bescheid weiß.

Kurz: So wie Offene Gesellschaften von Voraussetzungen leben, welche sie selbst nicht schaffen können, so trifft dies auch für autoritäre Gesellschaften zu: Sie leben folglich - direkt oder indirekt - von den Errungenschaften Offener Gesellschaften und nutzen jenes Wissen für ihre autokratischen Entscheidungen. Das Wagnis, sich auf autoritäre Experimente einzulassen, besteht somit nicht nur darin, dass wir die Kontrolle an eine nicht weiter kontrollierte Staatsmacht abgeben, sondern auch darin, dass ein Leben in autokratischen Gesellschaften eigentlich nur gelingt, wenn es auf Menschen und Institutionen zurückgreifen kann, welche eine freiheitliche Ordnung und einen kritischen Diskurs immer schon vorhalten und pflegen.

\footnotetext{
${ }^{9}$ Helliwell/Layard/Sachs, World Happiness Report, 2018, 28; Kwon/Adler, Social capital: Maturation of a Field of Research, Academy of Management Review 39 (2014), 412-422.

${ }^{10}$ Popper, The Open Society and Its Enemies. Part 1: The Spell of Plato, 1945; ders., The Open Society and Its Enemies. Part 2: The high tide of prophecy: Hegel, Marx and the aftermath, 1945.
} 


\section{Das China-Experiment}

Das China-Experiment ist hierzu ein anschauliches Beispiel. In der chinesischen Kultur gilt es als besondere Lernleistung, wenn es gelingt, den Meister zu kopieren. Je perfekter dieser Vorgang gelingt, umso größer sind die Lernkurve und das Ansehen der Person. Diese ,Copy and Paste'-Kultur führt zunächst dazu, dass derjenige, der kopiert, einen Vorsprung genießt, weil er die ganze Last der Arbeit, der Erfindung und Herstellung, des Versuchs und Irrtums und des Scheiterns vermeintlich umgehen kann und sich völlig auf die Kopie konzentrieren kann. Zudem beeindruckt das China-Experiment mit seinen Größenordnungen und der Geschwindigkeit, mit welcher Entscheidungen manchmal umgesetzt werden. Das Gesetz der großen Zahl überstimmt die vielen kleinen, notwendigen fehlerfreundlichen Schritte, die erforderlich sind, um richtige Entscheidungen zu treffen. ${ }^{11}$

Gleichzeitig gehen dabei aber eine Reihe von anderen Kulturleistungen verloren bzw. werden gar nicht erst ausgebildet: kritische Auseinandersetzungen, Fehlerfreundlichkeit, öffentliche Diskurse, individuelle Urteilskraft und autonomes Denken. Sie sind allesamt Eigenschaften, welche nur in einer Offenen Gesellschaft gedeihen können. Autokratische Systeme müssen sich auf Imitationen, Kopieren und Nachahmungsstrategien verlassen, weil ihnen die ursprünglichen Ergebnisse einer kritischen Urteilskraft immanent nicht zur Verfügung stehen. In einer unsicheren, komplexen und totalvernetzten Welt brauchen wir aber mehr an kritischem Denken und weniger an Kopieren, mehr an eigenständigem Denken als an Imitation, mehr an Freiheit und Autonomie als an Kontrolle und Allmachts- und Herrschaftswissen - dies vor allem deshalb, weil auch der autokratische Herrscher auf ein Wissen setzen muss, welches ihm nur in kritischer Urteilskraft zugänglich ist. Das bonum commune bleibt auch weiterhin ein unbestimmter Rechtsbegriff, der sich durch autokratisches Wissen immanent nicht hinreichend einlösen lässt. ${ }^{12}$ Jenes gibt ein Wissen vor, welches es so gar nicht hat. Auch wenn es derzeit so aussehen mag, dass autokratische Regime einen Wettbewerbsvorteil gegenüber Offenen Gesellschaften haben, so täuscht dieser Eindruck. Es gibt nichts Wirkmächtigeres als kritisches, reflexives Denken von Menschen, die sich in Freiheit auf den Weg machen ein Problem zu lösen. Die Kulturleistung des Kopierens setzt eine noch weitaus größere Kulturleistung voraus, nämlich jene der autonomen kritischen Kreativität in Freiheit. Wissenschaft und Fakten, Erziehung und Kunst, Kultur und Ingenieurleistungen sind allesamt ohne jene selbstverantwortete autonome Urteilskraft nicht denkbar. Das ist wohl der tiefere Grund, weshalb das China-Polen-Ungarn-Brasilien-Russland-Experiment auf mittlere Sicht die zweitbeste Lösung ist und die Offene Gesellschaft die bessere. ${ }^{13}$

Daraus folgt: Illiberale Demokratien, gelenkte Demokratien, Ein-Parteien-Demokratien sind nicht identisch mit dem Verständnis von Demokratie und Rechtsstaat in der Offenen Gesellschaft und verbleiben so immer autoritär, auch wenn sie einen ähnlichen Namen tragen. Sie stellen ein historisches Experiment dar, welches zu schnell und zu kurz gesprungen ist. Auch wenn sie auf den ersten Blick großartig klingen mögen, sind sie doch eine regressive Antwort auf die anstehenden Herausforderungen im 21. Jahrhundert. Offene Gesellschaften leben stattdessen von der Idee einer freiheitlichen Ord-

${ }^{11}$ Sommer, China First, Die Welt auf dem Weg ins chinesische Jahrhundert, 2019, 22 ff.

${ }^{12}$ Voßkuhle, Rechtsstaat und Demokratie, NJW 2018, 3154.

${ }^{13}$ Brunnhuber, Die Offene Gesellschaft. Ein Plädoyer für Freiheit und Ordnung, 2019, 170 ff. 
nung oder einer geordneten Form der Freiheit. Die folgende Abbildung zeigt die Zusammenhänge:

Abbildung 1: Offene Gesellschaften setzen auf Kreativität, Kritik und die Koexistenz von vielfältigen Lebensentwürfen; autokratische Ordnungen dagegen setzen auf Kopien, Konformismus und Kontrolle. Da jene auf die Ergebnisse der ersten angewiesen sind, bleiben sie selbstlimitierend und dependent.

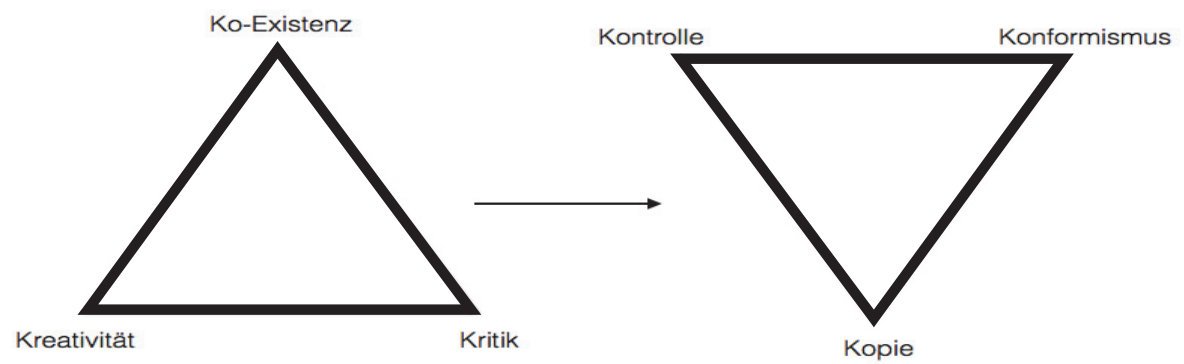

\section{Schluss oder können Offene Gesellschaften Nachhaltigkeit?}

Thomas Friedman ${ }^{14}$ hat vorgeschlagen, dass Amerika für einen Tag China sein solle, damit die allseits nötige Umweltpolitik und allfällige Innovationen leichter umgesetzt werden könnten. Damit wäre die schlechteste Seite der Demokratie umgangen - große Entscheidungen in Friedenszeiten zu treffen, um dann die besten Seiten der Demokratie - unsere Zivilgesellschaft, die Revision und die Implementierung von Gesetzen, freie Märkte, freie Presse, freie Mobilität und ein freier Wissenschaftsbetrieb - wieder hinreichend wertzuschätzen. Man kann dennoch fragen: Kann die Offene Gesellschaft mit ihrer Form der demokratischen Grundverfassung ,Nachhaltigkeit‘? Denn mit dem neuen Zeitalter des Anthropozäns ${ }^{15}$ ändert sich alles, auch das Verständnis von Demokratie, Rechtsstaatlichkeit und gesellschaftlicher Offenheit. Alle drei sind gegenüber den Herausforderungen des Anthropozäns nicht neutral. Es sind nicht nur die einzelnen politischen Programme, sondern auch die ihnen zugrundeliegende konstitutionelle Verfassung, welche über den Erfolg oder Misserfolg unseres Gemeinwesens im 21. Jahrhundert entscheiden. Scheitert also unser demokratisches System an der Umweltfrage und an der Organisation komplexer globaler Allgemeingüter - weil ihm nicht die ,policy“, also das politische Programm, sondern auch die ,polity', die konstitutionellen Voraussetzungen fehlen? Sind Demokratien zu langsam, zu schwerfällig und zu unbestimmt in der Frage, wer alles mitentscheiden soll - alle oder vor allem Experten oder

${ }^{14}$ Friedman, Was zu tun ist. Eine Agenda für das 21. Jahrhundert, (2008) 53.

${ }^{15}$ Crutzen, Geology of mankind, Nature, 415, (2002) 23. 
wer sonst noch? Und generieren sie vielleicht zu wenig relevantes und signifikantes Wissen und Information, um dann die richtigen Entscheidungen zu treffen (Fakes versus Fakten)? ${ }^{16}$ Die Fragen lassen sich nur durch eine Gegenfrage beantworten: Wer sonst? Denn die Alternative autokratischer Regime bleibt ein historisches Experiment, welches auf eine Kulturleistung zurückgreifen muss, die es nur in Offenen Gesellschaften gibt: Es gibt eben nichts Mächtigeres als freie Menschen, die selbstkritisch, autonom und im Wissen um ihre eigenen Grenzen und Fehlbarkeit in ständigen öffentlichen und zugleich angstfreien Diskursen um die Wahrheit, Freiheit und Ordnung ringen. Dies alles spricht nicht einmal gegen den Erfolg autokratischer Experimente in einzelnen Regionen der Welt, sondern nur für ihren selbstlimitierend und dependenten Charakter. Wenn wir allerdings davon ausgehen, dass wir schon alles wissen, dann sollten wir uns auf das autokratische Experiment einlassen.

Stefan Brunnhuber,

Hochschule Mittweida,

E-Mail: brunnhub@hs-mittweida.de

${ }^{16}$ Heidenreich, How will Sustainability transform Democracy, Reflexions on an important dimension of Transformation Sciences, Gaia, 27/4 (2018), 357-362. 